(Aus der Universitäts-Augenklinik zu Heidelberg. [Direktor: Geh. Hofrat Professor Dr. Wagenmann].)

\title{
Weitere experimentelle Untersuchungen über die Quelle und den Verlauf der intraokularen Saftströmung.
}

\author{
VI. Mitteilung. \\ Die Filtrationsfähigkeit, eine wesentliche Eigenschait der Scleralnarben \\ nach erfolgreicher Elliotscher Trepanation. \\ Von \\ Professor Dr. Erich Seidel, \\ Oberarzt der Klinik.
}

Bei Scleralnarben nach erfolgreicher, d. h. den Augendruck zur Norm zurückführender Elliotscher Trepanation läßt sich noch nach vielen Jahren die Filtrationsfähigkeit der Scleralnarbe durch einen einfachen Versuch dartun und prüfen:

Man tropfe auf die die Scleralnarbe deckende Bindehaut einen Tropfen einer 2 proz. Fluoreseeinka 'ium ösung.

In einer Reihe von Fällen sieht man, wie ich das bereits beschrieben und demonstriert habe ${ }^{1}$, an dem nach kurzer oder etwas ängerer Zeit erfolgenden Farbenumschlag der rotbraunen Fluoresceirlösung ins Gelblichgrüne, daß ein deutlicher Flüssigkeitsdurchtritt durch die die Scleralnarbe deckende Bindebaut hindurch erfolgt und zwar in einzelren Fällen in Form eines kontinuierlichen Stromes bei voller Sel schärfe und einem intraokularen Drucke von $15-18 \mathrm{~mm} \mathrm{Ho}$.

In vielen Fällen kann man jedoch, selbst bei längerem $\mathrm{Zu}$ warten nach erfolgter Fluoresceinauftropfung und bei Verwendung einer dünneren Fluoresceinlösung, keine deutliche Grünfärbung über dem mehr oder weniger ausgebildeten oder auch ganz fehlenden Bindehautkissen wahrnehmen.

tbt man jedoch in diesen Fällen nach Auftropfen von Fluorescein einen gelinden Druck auf den Bulbus aus, z. B. mit dem stumpfen Ende eines entfernt von der Sc'eralnarbe aufgesetzten G.asstäbchens, so sieht man regelnäßig nach kurzem Zeitintervall aus der die Scleralnarbe deckend en Bindehant mit Deutlichkeit Flüssigkeitsmengen diffus, wie aus einem Schwamm, herausquellen, was man an dem nunmehr sofort und sehr deutlich erfolgenden Farbenumschlag (infolge der ein-

1) Vgl. v. Graefes Arch. 102, Heft 3 u. 4, S. 372; - Ber. über die 42. Vers. d. Deutschen ophth. Gesellseh. Heidelberg 1920 (Schlußwort zu meinem Vortrage). 
tretenden Verdünnung der aufgetropften Fluoresceinlösung) und dem sich jetzt bildenden und sichtbar werdenden grünlichgelben Flüssigkeitsstrome erkennt, der sich vertikal nach unten ergießt.

Nach mehrfach wiederholter Anstellung des Versuches an demselben Auge kann man eine Vergrößerung eines vorher vorhandenen Bindehautkissens oder auch die Bildung eines solchen (vorher nicht wahrgenommenen) nachweisen. Der bei Beginn normale Augendruck wird nach mehrfach wiederholten Versuchen bei der tonometrischen Messung um einige Millimeter $\mathrm{Hg}$ geringer gefunden.

Der geschilderte Versuch gelingt nur dam, wenn die Elliotsche Trepanation die beabsichtigte druckherabsetzende Wirkung zur Folge gehabt hat.

Die Länge der seit der Operation verstrichenen Zeit spielt für das Gelingen des Versuches keine Rolle.

Die Versuche wurden ausgeführt an einer größeren Anzahl (über 40) schon vor Jahren trepanierten Augen. Darunter befanden sich mehrere, die bereits vor 7 Jahren trepaniert worden waren. Die bei der Operation benutzte Trepangröße betrug in allen Fällen $1,5 \mathrm{~mm}^{1}$ ).

Die allein mögliche Erklärung der geschilderten Beobachtungen ist folgende:

Die Scleralnarbe nach erfolgreicher, d. h. den Augendruck herabsetzender Elliotscher Trepanation ist in allen Fällen, selbst noch nach Jahren, für Kammerwasser durchgängig, d. h. sie ist porös. Es besteht also in diesen Fällen durch einen oder mehrere, wenn auch nur mikroskopische Kanäle eine Verbindung zwischen Vorderkammer und subconjunctivalem Gewebe. Man kann daher vom Vorhandensein einer Kammerwasserfistel oder Vorderkammerfistel sprechen.

In den Fällen, bei denen spontan nach Auftropfen von Fluorescein auf die Bindehaut nach kürzerer oder längerer Zeit ein Farbenumschlag ins Grüne oder sogar ein grüner Flüssigkeitsstrom sichtbar wird, besteht außerdem eine Kommunikation zwischen subconjunctivalem Gewebe und der Oberfläche der Bindehaut durch eine oder mehrere, wenn auch nur mikroskopische Poren, durch welche sich das aus der Vorderkammer stammende Kammerwasser auf die Oberfläche der Bindehaut spontan in nachweisbaren Mengen entleert. Da man bekanntlich physikalisch einen porösen Körper, durch den Flüssigkeit hindurchtritt, als Filter bezeichnet, und der Durehtritt von Flüssigkeit durch denselben Filtration genannt wird, so muB man die beschriebene klinische $\mathrm{Er}$ scheinung als einen Beweis für die Filtrationsfähigkeit der

1) Ich habe mit der geschilderten Untersuchungsmethode auch zwei Fälle nach erfolgreicher Sklerektomie (Lagrange) untersucht und eine deutliche Filtrationsfähigkeit der Narbe festgestellt. Der eine Fall war vor $10 \mathrm{Jahren}$ hier operiert worden. 
Wlliotsoheu Soleraluabe ansehen, zumal man in einer Reihe von Fällen beobachten kann, daß im Kammerwasser vorhandene ungelöste Substanzen (Pigmentkörnchen) gleichsam als Filterrückstand im episkleralen Narbengewebe zurückgehalten werden ${ }^{1}$ ).

In den Fälen, bei denen nach Auftropfen von Fluorescein auf die Bindehaut $z$ unächst spontan kein grüner Flüssigkeitsstrom sichtbar wird, sondern ein solcher erst nach gelindem Druck auf den Bulbus. zur Wahrnehmung gelangt, wird das spontan aus der Vorderkammer durch die poröse Scleralnarbe entleerte Kammerwasser im subconjunctivalen Gewebe so schnell resorbiert, daß für gewöhnlich der Druck des subconjunctivalen Kammerwassers nicht genügt, um die die Scleralnarbe deckende Bindehaut, die im. Einzelfalle von verschiedener Besehaffenheit sein kann, in einer mit obiger Untersuchungsmethode nachweisbaren Menge spontan zu durchdringen. Erst wenn wir den Filtrationsdruck und somit die Filtrationsgeschwindigkeit erhöhen durch gelinden Druck auf den Bulbus, der zu vermehrtem AusfluB von Kammerwasser durch die Poren der Scleralnarbe und somit zu einer Vergrößerung des subconjunctivalen Kissens führt, vermag das Kammerwasser infolge des erhöhten Filtrationsdruokes, sowie infolge der jetzt eingetretenen Bindehautdehnung durch die nunmehr erweiterten Poren. der Bindehaut ${ }^{2}$ ) in größerer. Menge auf ihre Oberfläche zu treten, wo wir es mit unserer Untersuchungsmethode als grünen Kammerwasserstrom wahrnehmen.

Die geschilderten Versuche erbringen den Beweis, daß die Filtrationsfähigkeit eine wesentliche Eigenschaft der Seleralnarben nach erfolgreicher, d. h. den Augendruck herabsetzender Elliotscher Trepanation ist ${ }^{3}$ ). Denn es wurde festgestellt:

1) Aus diesen Ausführungen geht hervor, daß unter den vorliegenden Verhältnissen die Bezeichnungen "Filtrationsfähigkeit" und ,fistu lierende Beschaffenheit" (der Skleralnarbe) zwei verschiedene Worte für denselben Begriff darstellen.

2) Daß eine Zunahme der Porengröße der die Scleralnarbe deckenden Bindehaut beim Zustandekommen der geschilderten Erscheinungen gelegentlich eine Rolle mitspielen kann, geht aus folgender Beobachtung hervor: Man beobachtet gelegentlich Fälle, bei denen zunächst spontan mit der geschilderten Untersuchungsmethode kein deutlicher Flüssigkeitsaustritt dureh die Bindehaut festzustellen ist. Wiederholt man jedoch den Versuch, nachdem man mehrere Male einengelinden Druck af den Bulbus ausgeübt $\mathrm{h} a \mathrm{t}$, so sieht man dann in diesen Fällen, dab nunmehr auch ohne Druck auf den Bulbus, also spontan, ein deutlicher Farbenumsehlag über dem jetzt etwas vergröBerten Bindehautkissen eintritt, d. $h$. daß also jetzt ein nachweisbarer Flüssigkeitsaustritt durch die Bindehaut hindurch spontan erfolgt.

3) Vgl. dazu die entgegengesetzten Anschauungen anderer Autoren, zusammengestcllt durch Hamburger: Errährung des Auges 1914, S. 105-114; sowie Klin. Monatsbl. f. Augenheilk. 65, 40ff. 1920 ; - Ber. d. 42. Vers. d. Deutschen ophth. Gesellsch. Heidelberg 1920. (Vortrag von Hamburger.) 
iber die Quelle und den Verlauf der intraoknlaren Saftstrümung. V1. 161

1. AaB die Filtrationsfähigkeit der Scleralnarbe mit obiger Untersuchungsmethode'nur dann nachweisbar war, wenn dic ausgeführte Elliotsche Trepanation die druckherabsetzende Wirkung auf das. Auge zur Folge gehabt hatte;

2. daß die Filtrationsfähigkeit der Scleralnarbe nur so lange nachweisbar war, als die druckherabsetzende Wirkung der Elliotschen Trepanation auf das Auge anhielt, wie ich in Fällen beobachtete, bei denen der ursprünglich durch die Operation herabgesetzte Augendruck allmählich wieder über die Norm anstieg bei gleichzeitigem Versiegen des zuerst mit der geschilderten Untersuchungsmethode spontan oder auf leichten Druck nachweisbaren Kammerwasserabflusses durch Scleralnarbe und Bindehaut.

Aus diesen Beobachtungen ergibt sich:

1. daB die druckherabsetzende Wirkung der Elliotschen Trepanation auf der Filtrationsfähigkeit der Scleralnarbe beruht ${ }^{1}$ ),

2. daßimglaukomatösen Auge die Höhedes Augendruckes und die Menge des in der Zeiteinheit abfließenden Kammerwassers in ursächlichem Zusammenhang stehen,

3. daß die intraokulare Drucksteigerung beim chronischen Glaukom auf einen für das betr. Auge ungenügenden AbfluB des Kammerwassers bezogen werden muB.

Wir gelangen also durch die geschilderten Untersuchungen genau zu dem gleichen Ergebnis, das wir auf Grund ganz und gar anderer in der vorigen Mitteilung ${ }^{2}$ ) geschilderten Beobachtungen a $\mathrm{m}$ gla ukomatösen Auge erhielten:

„Erleichterung des Abflusses führt zur Drucksenkung, Erschwerung des Abflusses zum Druckanstieg."

1) $\mathrm{Da}$ ich weiter mittels der geschilderten Untersuchungsmethode feststellte, daß nach erfolgreicher antiglaukomatöser Iridektomie (bei akutem und bei Sekundär-Glaukom) eine Filtrationsfähigkeit der Narbe nicht vorhanden ist, so ergibt sich, daB die druckherabsetzende Wirkung der v. Graefeschen Iridektomie (im Gegensatz zur Elliotschen Trepanation) nicht auf der Filtrationsfähigkeit der Narbe beruhen kann, sondern auf einem andern Prinzip beruhen $\mathrm{muB}$, worauf ich in einer der folgenden Mitteilungen zurückkommen werde.

2) v. Graefes Archiv f. Ophth. 102, 415-420. 